\title{
Positron stopping power in some biological compounds for intermediate energies with generalized oscillator strength model
}

\begin{abstract}
In this study total inelastic differential cross section and related stopping power expressions for positron were obtained by using generalized oscillator strength model. The stopping powers of low atomic number targets and of some biological compounds for positrons were calculated over the energy range from $50 \mathrm{eV}$ to $10 \mathrm{MeV}$. Calculations of the stopping powers (SPs) for some low atomic number targets: $\mathrm{H}_{2}, \mathrm{C}, \mathrm{N}_{2}, \mathrm{O}_{2} \mathrm{P}$ and for biological compounds: $\mathrm{C}_{5} \mathrm{H}_{5} \mathrm{~N}_{5}$ (adenine), $\mathrm{C}_{5} \mathrm{H}_{5} \mathrm{~N}_{5} \mathrm{O}$ (guanine), $\mathrm{C}_{5} \mathrm{H}_{5} \mathrm{~N}_{3} \mathrm{O}$ (cytosine), $\mathrm{C}_{5} \mathrm{H}_{6} \mathrm{~N}_{2} \mathrm{O}_{2}$ (thymine), $\mathrm{C}_{20} \mathrm{H}_{27} \mathrm{~N}_{7} \mathrm{O}_{13} \mathrm{P}_{2}$ (cytosine-guanine), and $\mathrm{C}_{19} \mathrm{H}_{26} \mathrm{~N}_{8} \mathrm{O}_{13} \mathrm{P}_{2}$ (thymine-adenine), have been evaluated for incident positrons in the $50 \mathrm{eV}-10 \mathrm{MeV}$ energy range. A detailed comparison of the calculated results with the other theoretical and experimental data for these target materials were presented. The calculated results of stopping powers for positrons in energy range from $50 \mathrm{eV}$ to $10 \mathrm{MeV}$ were found to be in good agreement to within $5 \%$ above $100 \mathrm{eV}$ energies with other theoretical results.
\end{abstract}

Keywords: positron stopping power, generalized oscillator strength, biological compounds, bragg rule
Volume 3 Issue 3 - 2018

\author{
Hasan Gumus,' Tuba Namdar,' Abdelouahab \\ Bentabet $^{2}$ \\ IDepartment of Physics, Faculty of Science and Arts, Ondokuz \\ Mayis University, Turkey \\ 2 LCVRN, Bordj Bou Arreridj University, Aleria
}

\begin{abstract}
Correspondence: Hasan Gumus, Department of Physics, Faculty of Science and Arts, Ondokuz Mayis University, 55। 39 Samsun, Turkey, Tel+903623I2 1919/506I, Fax+90 362457608 I, Email hgumus@omu.edu.tr
\end{abstract}

Received: January 26, 2018 | Published: May 01, 2018

\section{Introduction}

Stopping powers (SPs) of matter for positrons are important in wide variety of applications such as nuclear medicine, radiology, basic paticle physics, health physics, and radiation dosimeters design. Positron stopping powers (PSPs) at energies above $10 \mathrm{keV}$ are theoretically well described and can be found in tables given in Berger \& Seltzer $^{1}$ and the ICRU 37 Report. $^{2}$ The stopping power formula for positrons obtained by Batra $^{3}$ had been fitted by a twoparameter approximation and is valid for the energy range from $1 \mathrm{keV}$ to $500 \mathrm{keV}$. On the other hand, Meiring et al., ${ }^{4}$ developed a theory of multiple scattering, exhibiting di $\square$ erences between positrons and electrons in the interaction with matter for the $\mathrm{MeV}$ energy range. In recent years there have been many studies on positron stopping power and its applications. ${ }^{5-8}$

We obtained, in a previous study ${ }^{9}$ the SP formula for intermediate energy electrons, by using the generalized oscillator strength (GOS) model. Stopping power Calculations for positrons have not been studied as much, though their tracks in matter are frequently assumed to be similar to those of electrons. Positrons are used for imaging purposes (for example, PET), but can also be used for cancer therapy. ${ }^{10}$ Hence, it is important to obtain SP values, for many applications in the lower energy range $(<1 \mathrm{keV})$. The purpose of this study is actually to obtain a SP formula for incident positrons, valid for the low and intermediate energy region $(<10 \mathrm{keV})$. The PSP formula given in this study is based on the Generalized Oscillator Strength (GOS) model ${ }^{11}$ and a modification of it using the e $\square$ ective electron number (EEN) and effective mean excitation energies (EMEEs). In this PSP formula, analytical expressions obtained in the previous work ${ }^{12,13}$ for the EEN and EMEE of target atoms are used. Thus the obtained calculating procedure was applied to evaluate the stppping power (SP) values for incoming positrons on hydrogen, carbon, nirogen, phospore, and liquid water targets. For positrons at low energies the inelastic interaction characteristics, stopping power, can not be obtained directly from Bethe's PSP theory or from experiments, which gives accurate PSPs at energies larger than $10 \mathrm{keV}$. At lower energies the theory is, in general, invalid. For low energy positrons a method used way to estimate the mentioned characteristic are dielectric theoretical methods, based on the use of the complex dielectric function $\varepsilon(q, \omega), m$ and $m$ being the momentum and energy transfer. To calculate the stopping power, other method is to make use of the inelastic differential cross section suggested by Inokuti ${ }^{11}$ with Generalized Oscillator Strength (GOS). Actulally, the GOS model has to be calculated from matrix elements that involve numerical integration of atomic wave functions. The aim of this study is to obtain stopping power and total inelastic differential cross section expressions for positron using the GOS model for biological compounds, valid for low and intermediate energies. Applying the GOS model, anlytical expressions for the calculation of stopping Powers (SPs) have been given for biological targets. In this study total inelastic differential cross sections and stopping power expressions for positron were obtained by using Generalized Oscillator Strength (GOS) model. The calculated results of PSPs of the biological compounds, have been compared with the theoretical and semiemprical results. In additon, using the described model in this study, the PSPs for some important biological compounds $\left(\mathrm{C}_{5} \mathrm{H}_{5} \mathrm{~N}_{5}\right.$ (adenine), $\mathrm{C}_{5} \mathrm{H}_{5} \mathrm{~N}_{3} \mathrm{O}$ (cytosine), $\mathrm{C}_{20} \mathrm{H}_{27} \mathrm{~N}_{7} \mathrm{O}_{13} \mathrm{P}_{2}$ (cytosine-guanine), $\mathrm{C}_{5} \mathrm{H}_{5} \mathrm{~N}_{5} \mathrm{O}$ (guanine), $\mathrm{C}_{5} \mathrm{H}_{6} \mathrm{~N}_{2} \mathrm{O}_{2}$ (thymine), and $\mathrm{C}_{19} \mathrm{H}_{26} \mathrm{~N}_{8} \mathrm{O}_{13} \mathrm{P}_{2}$ (thymine-adenine)) have been calculated (Table 1).

\section{Theory}

In inelastic interactions the target atom is either excited to a suitable higher level than the ground level or it is ionized depending on the energy that the arriving particle imparts to the target atom. 
Table I SPs $\left(\mathrm{MeVcm} / \mathrm{g}\right.$ ) for $\mathrm{I}_{-} \mathrm{C}_{5} \mathrm{H}_{5} \mathrm{~N}_{5}$ (adenine), 2- $\mathrm{C}_{4} \mathrm{H}_{5} \mathrm{~N}_{3} \mathrm{O}$ (cytosine), 3- $\mathrm{C}_{5} \mathrm{H}_{5} \mathrm{~N}_{5} \mathrm{O}$ (guanine), 4- $\mathrm{C}_{5} \mathrm{H}_{6} \mathrm{~N}_{2} \mathrm{O}_{2}$ (thymine), 5- $\mathrm{C}_{20} \mathrm{H}_{27} \mathrm{~N}_{7} \mathrm{O}$ 13 $\mathrm{P}_{2}$ (cytosine-guanine), 6- $\mathrm{C}_{19} \mathrm{H}_{26} \mathrm{~N}_{8} \mathrm{O}_{13} \mathrm{P}_{2}$ (thymine-adenine) and 7- $\mathrm{H}_{2} \mathrm{O}$ (liquid water). Bold figures are the data of lower accuracy.

\begin{tabular}{|c|c|c|c|c|c|c|c|}
\hline Enerji (eV) & I- & $2-$ & 3- & 4- & 5- & 6- & 7- \\
\hline & Adenine & Cytosine & Guanine & Thymine & cytosine-guanine & thymine-adenine & Liquid water \\
\hline 50 & 608.44 & 551.57 & 544.02 & 503.54 & 425.85 & 429.94 & 287.54 \\
\hline 60 & 596.07 & 598.07 & 577.46 & 594.73 & 549.58 & 553.25 & 639.45 \\
\hline 70 & 575.44 & 578.56 & 559.63 & 576.07 & 534.1 & 537.32 & 625.72 \\
\hline 80 & 552.43 & 556.21 & 538.69 & 554.32 & 515.12 & 517.99 & 606.35 \\
\hline 90 & 529.39 & 533.59 & 517.27 & 532.13 & 495.35 & 497.93 & 585.1 \\
\hline 100 & 507.34 & 511.78 & 496.48 & 510.64 & 475.98 & 478.34 & 563.74 \\
\hline 200 & 355.27 & 359.69 & 350.05 & 359.71 & 337.24 & 338.52 & 404.08 \\
\hline 300 & 276.5 & 280.31 & 273.11 & 280.55 & 267.64 & 268.54 & 317.09 \\
\hline 400 & 228.49 & 231.81 & 225.99 & 232.12 & 223.39 & 224.1 & 263.22 \\
\hline 500 & 195.92 & 198.85 & 193.94 & 199.17 & $|92.4|$ & 192.99 & 226.36 \\
\hline 600 & $|72.2|$ & 174.84 & 170.58 & 175.16 & 169.67 & 170.17 & 199.38 \\
\hline 700 & I54.I & 156.5 & $|52.7|$ & $|56.8|$ & $|52.2|$ & 152.64 & |78.7| \\
\hline 800 & | 44.37 & 146.46 & 142.68 & |47.2| & 142.05 & 142.64 & 162.29 \\
\hline 900 & 132.89 & 134.8 & 131.32 & 135.56 & 130.85 & $|3| .4 \mid$ & $|48.9|$ \\
\hline 1000 & 123.26 & 125.03 & 121.8 & 125.79 & 121.46 & 121.99 & 137.76 \\
\hline 2000 & 76.75 & 77.88 & 76.17 & 78.27 & 75.96 & 76.19 & 86.68 \\
\hline 3000 & 56.64 & 57.46 & 56.25 & 57.73 & 56.1 & 56.27 & 63.77 \\
\hline 4000 & 45.44 & 46.08 & 45.13 & 46.3 & 45.02 & 45.15 & 51.06 \\
\hline 5000 & 38.21 & 38.74 & 37.96 & 38.92 & 37,94 & 38,05 & 42,88 \\
\hline 6000 & 33,12 & 33.58 & 32.91 & 33.74 & 32,91 & 33,00 & 37,15 \\
\hline 7000 & 29,33 & 29.74 & 29.15 & 29.88 & 29,16 & 29,24 & 32,87 \\
\hline 8000 & 26,39 & 26,75 & 26,23 & 26.88 & 26.24 & 26.31 & 29.55 \\
\hline 9000 & 24.04 & 24.37 & 23.9 & 24.48 & 23.91 & 23.97 & 26.9 \\
\hline 10000 & 22.11 & 22.41 & 21.99 & 22.51 & 21.1 & 22.05 & 24.73 \\
\hline 20000 & 12.73 & 12.9 & 12.66 & 12.96 & 12.68 & |2.7| & 14.2 \\
\hline 30000 & 9.25 & 9.37 & 9.2 & 9.41386 & 9.22 & 9.24 & 10.31 \\
\hline 40000 & 7.41 & 7.5 & 7.37 & 7.53742 & 7.39 & 7.41 & 8.25 \\
\hline 50000 & 6.25 & 6.33 & 6.22 & 6.35917 & 6.24 & 6.25 & 6.96 \\
\hline 60000 & 5.46 & 5.53 & 5.43 & 5.55659 & 5.45 & 5.46 & 6.07 \\
\hline 70000 & 4.89 & 4.95 & 4.86 & 4.97273 & 4.88 & 4.89 & 5.44 \\
\hline 80000 & 4.45 & 4.51 & 4.43 & 4.5264 & 4.44 & 4.45 & 4.95 \\
\hline 90000 & 4.1 & 4.16 & 4.08 & 4.18 & 4.1 & 4.11 & 4.56 \\
\hline 100000 & 3.83 & 3.87 & 3.81 & 3.89 & 3.82 & 3.83 & 4.25 \\
\hline 200000 & 2.54 & 2.57 & 2.53 & 2.58 & 2.54 & 2.54 & 2.82 \\
\hline 300000 & 2.12 & 2.14 & 2.11 & 2.15 & 2.12 & 2.12 & 2.35 \\
\hline 400000 & 1.92 & 1.94 & 1.91 & 1.95 & 1.92 & 1.92 & 2.13 \\
\hline 500000 & 1.81 & 1.83 & 1.8 & 1.84 & 1.8 & 1.81 & 2 \\
\hline 600000 & 1.74 & 1.76 & 1.73 & 1.77 & 1.74 & 1.74 & 1.93 \\
\hline 700000 & 1.7 & 1.72 & 1.69 & 1.73 & 1.7 & 1.7 & 1.89 \\
\hline 800000 & 1.67 & 1.69 & 1.66 & 1.7 & 1.67 & 1.68 & 1.89 \\
\hline 900000 & 1.66 & 1.68 & 1.65 & 1.68 & 1.66 & 1.66 & 1.83 \\
\hline 1000000 & 1.65 & 1.67 & 1.63 & 1.67 & 1.65 & 1.65 & 1.82 \\
\hline
\end{tabular}


Consider a positron (mass $m$, charge $+e$ ), moving with velocity $v$. IDCS) obtained from the first Born approximation is given by Inokuti ${ }^{11} \&$ Bichsel $^{14}$ as

$$
\frac{d^{2} \sigma}{d Q d W}=\chi \frac{1}{Q W} \frac{d f(Q, W)}{d W}
$$

where $\chi=2 \pi e^{4} / m c^{2} \beta^{2}, W$ is energy loss and $Q$ is the recoil energy given as

$$
Q=q^{2} / 2 m=2 E-W-2 \sqrt{E(E-W)} \cos \theta .
$$

$\mathrm{Q}$ is normally used instead of the momentum transfer $\boldsymbol{q}$ or the angular deflection $\theta ; E$ is the kinetic energy of the incident particle. The function $d f(Q, W) / d W$ is the genralized oscillator strength (GOS), which is described in detail by Inokuti. ${ }^{11}$ The GOS can be represented as a surface over the plane $(Q, W)$, known as the Bethe surface, is defined as

$$
\frac{d f(Q, W)}{d W} \equiv \frac{W}{Q} \mid\left.\langle\Psi| \sum_{j=1}^{Z} \exp \left(\text { iq. } r_{j} / \hbar\right)\left|\Psi_{0}\right\rangle\right|^{2}
$$

where $\Psi_{0}$ and $\Psi$ are the ground state and excited state atomic or molecular wave functions, respectively, and the sum runs over the $Z$ electrons in the target.

The total IDCS can be written as follows. ${ }^{15}$

$$
\sigma^{n}=\int_{0}^{W_{\max }} d W W^{n} \int_{Q_{-}}^{Q_{+}} d Q \frac{d^{2} \sigma}{d Q d W}
$$

The recoil energies lie in the interval $Q_{-}<Q<Q_{+}$and $Q_{-}$, is given by Fano $^{16}$ as mentioned by Inokuti ${ }^{17}$ as follows:

$$
Q_{-}(E, W)=\sqrt{\left(m c^{2}\right)^{2}+\frac{W^{2}}{\beta^{2}}}-m c^{2}
$$

The stopping power is given as follows:

$$
S=N \sigma^{(1)}
$$

Heree $\sigma^{(1)}$ is the stopping cross section. The function $d f(Q, W) / d W$ is the GOS. This GOS satisfies the Bethe sum rule:"1

$$
\frac{1}{Z} \int_{0}^{\infty} \frac{d f(Q, W)}{d W} d W=1 \quad \text { or } \sum_{i=1}^{M} f_{i}=Z
$$

Here $Z$ is the number of electrons per atom or molecule. The GOS is given by Liljequist ${ }^{18}$ as

$$
\frac{d f(Q, W)}{d W} \equiv \sum_{i=1}^{M} f_{i} F\left(W_{i} ; Q, W\right)
$$

where $M, f_{i}$ and $W_{i}$ are the number of shells, oscillator strengths for the $i$ th shell of the target atom and $i$ th shell resonance energies, respectively. Oscillators may be considered as the total spectrum of excitations of electrons belonging to a shell. The excitation spectrum, $F\left(W_{i} ; Q, W\right)$ has been suggested by Liljequist ${ }^{18}$ to be written as

$$
F\left(W_{i} ; Q, W\right)=\delta\left(W-W_{i}\right) \theta\left(W_{i}-Q\right)+\delta(W-Q) \theta\left(Q-W_{i}\right)
$$

where $\delta(x)$ is the Dirac delta function and $\theta(x)$ is a step function $(\theta(x)=0$ if $x<0$ and $\theta(x)=1$ if $x \geq 0)$.
According to Bohr ${ }^{19}$ and as mentioned by Liljequist, ${ }^{18}$ the inelastic excitations may be separated into two parts because of resonance-like interactions with bound electrons (inner shell) and other interactions with large momentum transfer where the atomic electrons may be considered as free. The resonance-like interactions correspond to excitations with low momentum transfer $(Q\langle W)$ and with energy transfers of the order of the binding energy $\left(W=W_{i}\right)$. Interactions with large momentum transfer are defined as $Q \rightarrow 0$; in this region the atomic electrons are considered as rest and free.

The corresponding the OOS reduces to

$$
Q \rightarrow 0 \frac{d f(Q=0, W)}{d W}=\frac{d f(W)}{d W}
$$

Preparation of the OOS for inner shell ionization (generally $\mathrm{K}$ shell) and for energy losses above $\approx 100 \mathrm{eV}$, has been obtained either from optical data ${ }^{11}$ or by data in the X-ray region related to inner shell ionization. In this situation, the OOS is calculated by means of the relation. ${ }^{15,20}$

$$
\frac{d f(W)}{d W}=\frac{m c}{2 \pi^{2} \hbar e^{2}} \sigma_{p h} \text { or } f_{k}=\frac{1}{109.8} \int_{B_{k}}^{\infty} \sigma_{p h}(E) d E
$$

where $\sigma_{p h}(E)$ is the photoelectric cross section (in barns) at a given energy $E$ (in $\mathrm{MeV}$ ). The photoelectric cross section, $\sigma_{p h}$, can be obtained from experimentally based tables ${ }^{21}$ or by using theoretical photoelectric cross section formulae. Other than this is a Local Plasma Approximation (LPA) of the OOS. ${ }^{22}$ For several molecules, the K shell oscillator strength, $f_{k}$ was calculated by means of a numerical integration (trapezoidal rule) of Eq. (11) using photoelectric cross sections given by Henke et al., ${ }^{21}$ at binding energies from $50 \mathrm{eV}$ to $1 \mathrm{keV}$ and for other energies $(1 \mathrm{keV}$ to $1 \mathrm{MeV})$ using the XCOM program produced Berger et al., ${ }^{23}$ by Akar \& Gumus., ${ }^{9}$ In this paper, with the aim of checking of OOSs, we used the values of $a, f_{\mathrm{k}}$ and $W_{\mathrm{v}}$ callculated $b$ Akar \& Gumus ${ }^{9}$ and culcated he alues of $f$ and for biological targets. $I$ for positrons. The mean ionisation energy of biological compounds were calculated from Bragg's addition rule. ${ }^{24}$

\section{Theoretical calculation of positron stopping powers}

Excitations with $Q=W$ which have resonance-like character can be defined as distant collisions and excitations with $Q=W$ which correspond to free collisions may be referred to as close collisions. ${ }^{24}$ The DCS for inelastic collisions obtained from GOS model can be split into contributions from distant longitudinal, distant transverse and close ineraction,

$$
\frac{d^{2} \sigma_{i n}}{d Q d W}=\frac{d^{2} \sigma_{d, l}}{d Q d W}+\frac{d^{2} \sigma_{d, t}}{d Q d W}+\frac{d^{2} \sigma_{c}}{d Q d W}
$$

For the case distant interactions, the CSs of electrons and positrons are the same. But positrons in matter are unstable particle that annihilate with s giving photons. Electron-positron pairs can be created if enough electromagnetic energy $\left(>2 \mathrm{mc}^{2}\right)$ is available (either from real or virtual photons). A positron does not interact with matter as a usual stable positively charged particle, since the competing process of annihilation followed by re-creation can cause the same transitions as "direct" scattering. ${ }^{25,26}$ The DCS for binary collisions of positrons with free electrons at rest, obtained from the first Born approximation including the "annihilation or creation" mechanism, is given by the Bhabha ${ }^{27}$ formula, 
$\frac{d^{2} \sigma_{B}}{d Q d W}=\chi \frac{1}{W^{2}}\left[1-b_{1} \frac{W}{E}+b_{2}\left(\frac{W}{E}\right)^{2}-b_{3}\left(\frac{W}{E}\right)^{3}+b_{4}\left(\frac{W}{E}\right)^{4}\right] \delta(W-Q)$

where

$$
\begin{aligned}
& b_{1}=\left(\frac{\gamma-1}{\gamma}\right)^{2} \frac{2(\gamma+1)^{2}-1}{\gamma^{2}-1} \\
& b_{2}=\left(\frac{\gamma-1}{\gamma}\right)^{2} \frac{3(\gamma+1)^{2}+1}{\left(\gamma^{2}-1\right)^{2}}
\end{aligned}
$$

$$
\begin{aligned}
& b_{3}=\left(\frac{\gamma-1}{\gamma}\right)^{2} \frac{2 \gamma(\gamma-1)}{\left(\gamma^{2}+1\right)^{2}} \\
& b_{2}=\left(\frac{\gamma-1}{\gamma}\right)^{2} \frac{(\gamma-1)^{2}}{(\gamma+1)^{2}}
\end{aligned}
$$

The DCSs for distant interactions is the sum of contributions from transverse and longitudinal interactions:

$$
\frac{d \sigma_{d}}{d W}=\chi \sum_{k} f_{k}\left\{\frac{1}{W} \frac{2 m_{e} c^{2}}{Q\left(Q+2 m_{e} c^{2}\right)}+\frac{1}{W}\left[\ln \left(\frac{1}{1-\beta^{2}}\right)-\beta^{2}-\delta_{F}\right]\right\} \delta\left(E-W_{k}\right) \theta\left(W_{k}-Q\right)
$$

Using Eq. (13) and Eq. (14), total inelastic differential cross section are given with following expression: $\sigma_{i n}^{(1)}=\chi$

$\sum_{k} f_{k}\left\{\ln \left(\frac{W_{k} Q_{-}+2 m_{e} c^{2}}{Q_{-} W_{k}+2 m_{e} c^{2}}\right)+\ln \left(\frac{1}{1-\beta^{2}}\right)-\beta^{2}-\delta_{F}+\frac{1}{W}\left[1-b_{1} \frac{W}{E}+b_{2}\left(\frac{W}{E}\right)^{2}-b_{3}\left(\frac{W}{E}\right)^{3}+b_{4}\left(\frac{W}{E}\right)^{4}\right]\right\} \Theta\left(W_{m a k}-W_{k}\right)$

By using Eq. (6) and Eq. (16), and taking $W_{\max }=E$, SP for positrons can be written as follow,

$$
\begin{aligned}
& S(E)=N \chi \\
& \sum_{k} f_{k}\left\{\ln \left(\frac{W_{k} Q_{-}+2 m_{e} c^{2}}{Q_{-} W_{k}+2 m_{e} c^{2}}\right)+\ln \left(\frac{1}{1-\beta^{2}}\right)-\beta^{2}-\delta_{F}+\frac{1}{W}\left[1-b_{1} \frac{W}{E}+b_{2}\left(\frac{W}{E}\right)^{2}-b_{3}\left(\frac{W}{E}\right)^{3}+b_{4}\left(\frac{W}{E}\right)^{4}\right]\right\} \Theta\left(W_{\text {mak }}-W_{,}\right)
\end{aligned}
$$

For positrons the SP of compounds were calculated from Bragg's addition rule ${ }^{24}$ as follows:

$$
\frac{\mathrm{S}(\mathrm{E})}{\rho} \text { (compound) }=\sum_{i} w_{i}\left[\frac{S(E)}{\rho}\right]_{i}
$$

where $w_{i}$ are the atomic fractions in molecule.

\section{Calculated results and discussion}

In this study, to obtain the inelastic SPs for positrons of biological materials the OS of the inner and valence shell PMIPs, $W_{i}$ and $W_{v}$, calculated by Akar \& Gumus ${ }^{9}$ were used in Eq. (17). The calculated PSPs for $\mathrm{H}_{2}, \mathrm{C}, \mathrm{N}_{2}, \mathrm{O}_{2}, \mathrm{P}, \mathrm{C}_{5} \mathrm{H}_{5} \mathrm{~N}_{5}$ (adenine), $\mathrm{C}_{4} \mathrm{H}_{5} \mathrm{~N}_{3} \mathrm{O}$ (cytosine), $\mathrm{C}_{20} \mathrm{H}_{27} \mathrm{~N}_{7} \mathrm{O}_{13} \mathrm{P}_{2}$ (cytosine-guanine), $\mathrm{C}_{5} \mathrm{H}_{5} \mathrm{~N}_{5} \mathrm{O}$ (guanine), $\mathrm{C}_{5} \mathrm{H}_{6} \mathrm{~N}_{2} \mathrm{O}_{2}$ (thymine), $\mathrm{C}_{19} \mathrm{H}_{26} \mathrm{~N}_{8} \mathrm{O}_{13} \mathrm{P}_{2}$ (thymine-adenine) and liquis warer are given in the Figs. 1-12.

Figure 1 shows the mass stopping power (mSP) for incident positrons from $50 \mathrm{eV}$ to $10 \mathrm{MeV}$ energy range in hydrogen. The results obtained from this study are compared with the PENELOPE program $^{26}$ and results for positrons by using Gumus calculation methods. ${ }^{12,28}$ The calculated PSPs values obtained by using Eqs. (17, 18) are good agreement with PENELOPE program ${ }^{26}$ results to within $1 \%$ expect at energies below $100 \mathrm{eV}$.

Figure 2 shows the mSP for incident positrons from $50 \mathrm{eV}$ to 10 $\mathrm{MeV}$ energy range in carbon. The results obtained from this study are compared with the PENELOPE program ${ }^{26}$ values of ICRU 37 report and results for positrons by using Gumus calculation methods. ${ }^{12,28}$ The calculated PSPs values obtained by using Eqs. $(21,22)$ are good agreement with PENELOPE program ${ }^{26}$ results to within $1 \%$. expect at energies below $300 \mathrm{eV}$.

Figure 3 shows the mSP for incident positrons from $50 \mathrm{eV}$ to 10 $\mathrm{MeV}$ energy range in nitrogen. The results obtained from this study are compared with the PENELOPE program. ${ }^{26}$ and results for positrons by using Gümüş calculation methods. ${ }^{12,28}$ The calculated PSPs values obtained by using Eqs. $(21,22)$ are good agreement with PENELOPE program 26 results to within $3 \%$.

Figure 4 shows the mSP for incident positrons from $50 \mathrm{eV}$ to 10 $\mathrm{MeV}$ energy range in oxygen. The results obtained from this study are compared with the PENELOPE program and results for positrons by using Gumus calculation methods. ${ }^{12,28}$ The calculated PSPs values obtained by using Eqs. $(17,18)$ are good agreement with PENELOPE program 26 results to within $3 \%$. 
Figure 5 shows the mSP for incident positrons from $50 \mathrm{eV}$ to 10 $\mathrm{MeV}$ energy range in phosphorus. The results obtained from this study are compared with the PENELOPE program 26 and results for positrons by using Gümüş calculation methods. ${ }^{8,29}$ The calculated PSPs values obtained by using Eqs. $(17,18)$ are good agreement with PENELOPE program ${ }^{22}$ results to within $2 \%$. expect at energies below $300 \mathrm{eV}$.

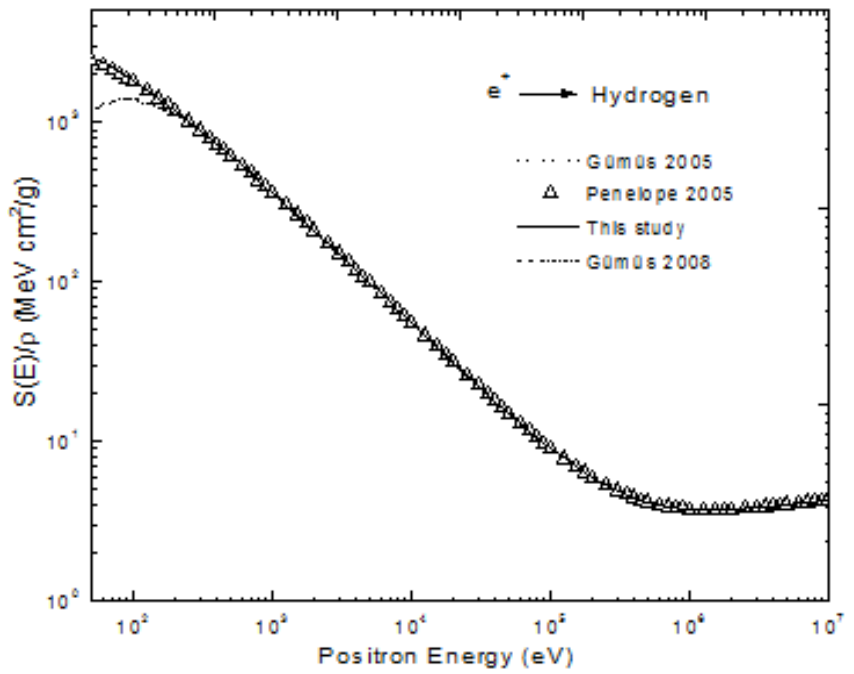

Figure I Mass stopping power $\mathrm{S}(\mathrm{E}) / \rho$ for incident positron energies, in $\mathrm{H}$. —, present study; ..., theoretical data from Gumus'2; and ----, from Gumus ${ }^{29}$ model; $\Delta$, results calculated by PENELOPE Program. ${ }^{27}$

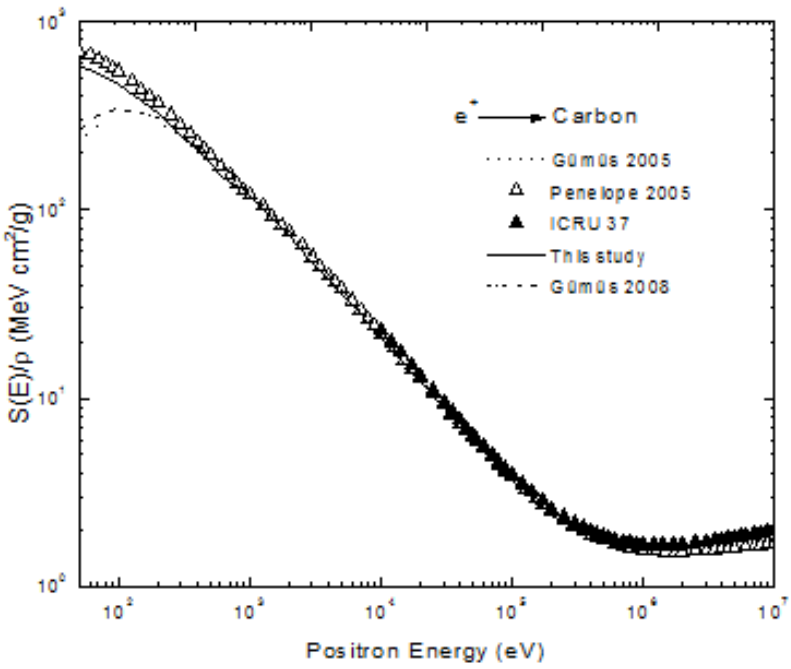

Figure $2 \mathrm{~S}(\mathrm{E}) / \rho$ for incident positron energies, in carbon. -, present study; ..., theoretical data from Gumus ${ }^{2}$; and ----, from Gumus ${ }^{29}$ model; $\Delta$, results calculated by PENELOPE Program; ${ }^{27} \boldsymbol{\Lambda}$, data from ICRU 37 report (I984).

Figure 6 shows the mSP for incident positrons from $50 \mathrm{eV}$ to 10 $\mathrm{MeV}$ energy range in adenin. The results obtained from this study are compared with the PENELOPE program. ${ }^{26}$ The calculated PSPs values obtained by using Eqs. $(17,18)$ are good agreement with PENELOPE program ${ }^{26}$ results to within $2 \%$.

Figure 7 shows the mSP for incident positrons from $50 \mathrm{eV}$ to 10 $\mathrm{MeV}$ energy range in cytosine. The results obtained from this study are compared with the PENELOPE program. ${ }^{26}$ The calculated PSPs values obtained by using Eqs. $(17,18)$ are good agreement with PENELOPE program results to within 3\%.

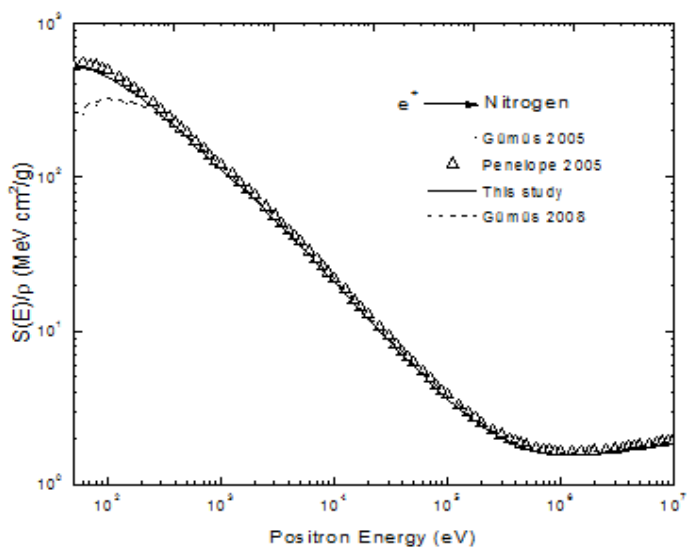

Figure $3 \mathrm{~S}(\mathrm{E}) / \rho$ for incident positron energies, in nitrogen;-—, present study; $\ldots$, theoretical data from Gumus' ${ }^{12}$; and ----, from Gumus ${ }^{29}$ model; $\Delta$, results calculated by PENELOPE Program. ${ }^{27}$

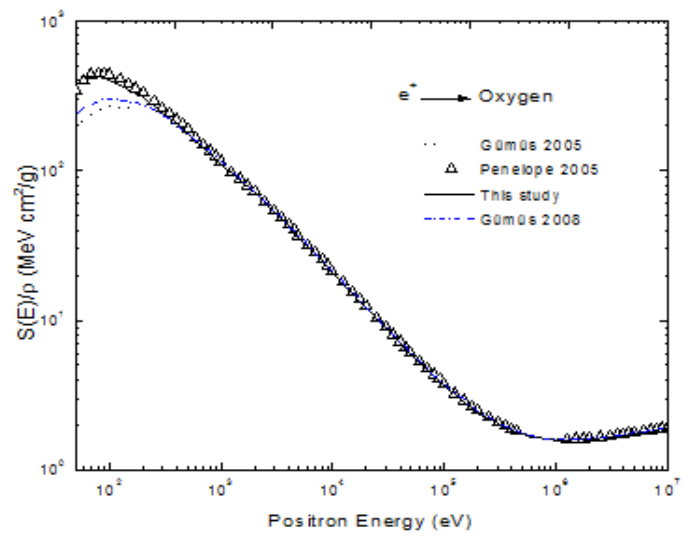

Figure $4 \mathrm{~S}(\mathrm{E}) / \rho$ for incident positron energies, in oxygen. -, present study; $\Delta$, results calculated by PENELOPE Program; ${ }^{27}$.... theoretical data of Gumus ${ }^{12}$ and ---- theoretical data of Gumus. ${ }^{29}$

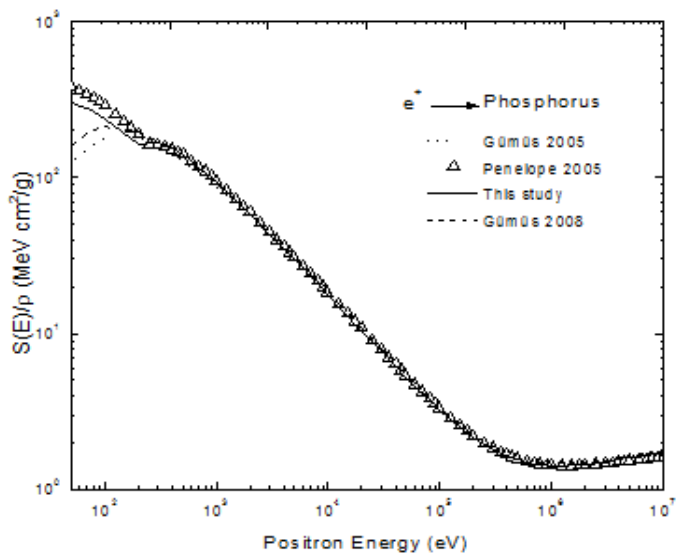

Figure $5 \mathrm{~S}(\mathrm{E}) / \rho$ for incident positron energies, in phosphorus. —, present study; $\Delta$, results calculated by PENELOPE Program; ${ }^{27}$.... theoretical data of Gumus ${ }^{8}$; and ---- theoretical data of Gumus. ${ }^{29}$ 


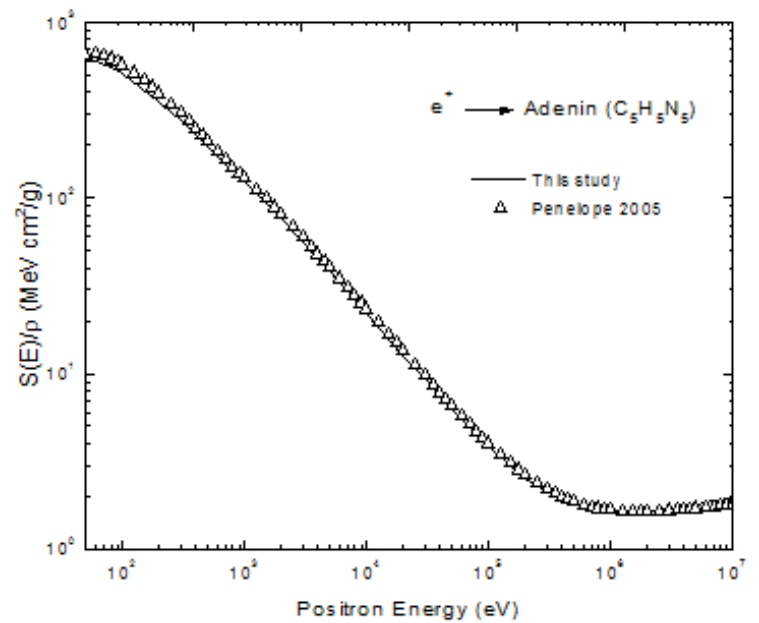

Figure $6 \mathrm{~S}(\mathrm{E}) / \rho$ for incident positron energies, in adenine. - , present study; $\Delta$, results calculated by PENELOPE Program. ${ }^{27}$

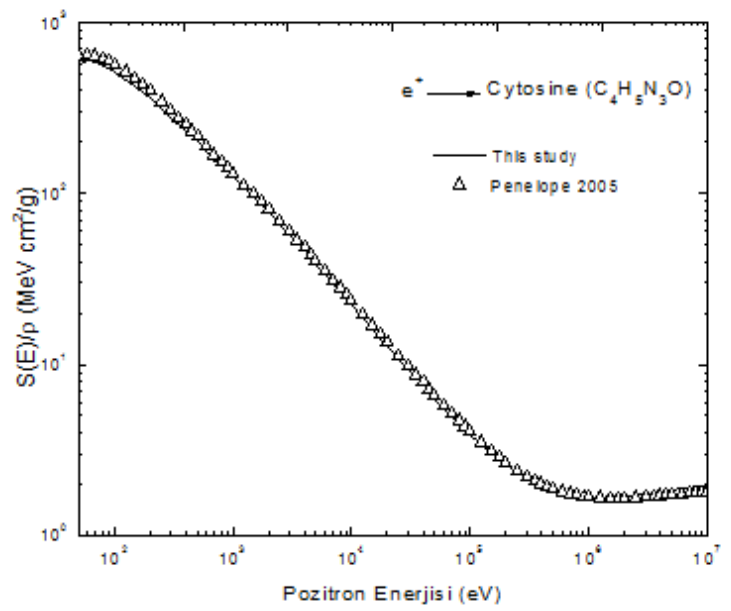

Figure $7 \mathrm{~S}(\mathrm{E}) / \rho$ for incident positron energies, in cytosine. - , present study; and $\Delta$, PENELOPE Program results.

Figure 8 shows the mSP for incident positrons from $50 \mathrm{eV}$ to 10 $\mathrm{MeV}$ energy range in guanine. The results obtained from this study are compared with the PENELOPE program..$^{26}$ The calculated PSPs values obtained by using Eqs. $(17,18)$ are good agreement with PENELOPE program results to within $4 \%$.

Figure 9 shows the mSP for incident positrons from $50 \mathrm{eV}$ to 10 $\mathrm{MeV}$ energy range in thymine. The results obtained from this study are compared with the PENELOPE program. ${ }^{22}$ The calculated PSPs values obtained by using Eqs. $(17,18)$ are good agreement with PENELOPE program results to within 5\%.

Figure 10 shows the mSP for incident positrons from $50 \mathrm{eV}$ to 10 $\mathrm{MeV}$ energy range in cytosine-guanine. The results obtained from this study are compared with the PENELOPE program. ${ }^{26}$ The calculated PSPs values obtained by using Eqs. $(17,18)$ are good agreement with PENELOPE program results to within 5\%.

Figure 11 shows the mSP for incident positrons from $50 \mathrm{eV}$ to 10 $\mathrm{MeV}$ energy range in thymine-adenine. The results obtained from this study are compared with the PENELOPE program.$^{26}$ The calculated PSPs values obtained by using Eqs. $(17,18)$ are good agreement with PENELOPE program results to within 5\%.

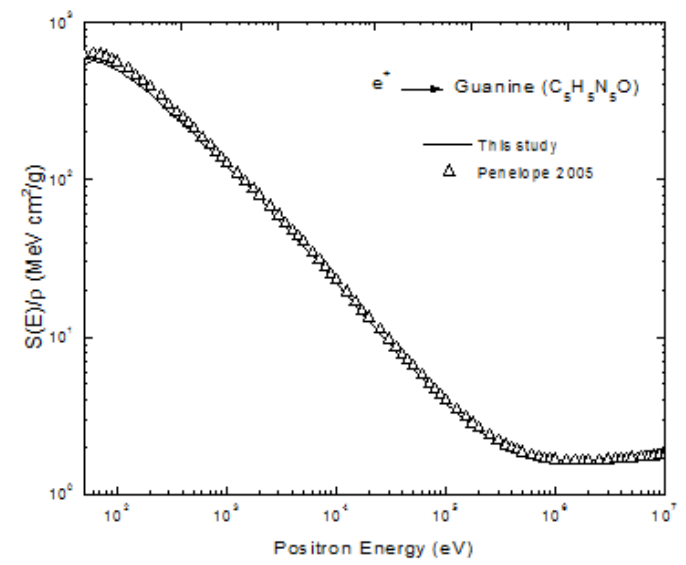

Figure $8 \mathrm{~S}(\mathrm{E}) / \rho$ for incident positron energies, in guanine. - , present study; and $\triangle$, PENELOPE Program ${ }^{27}$ results.

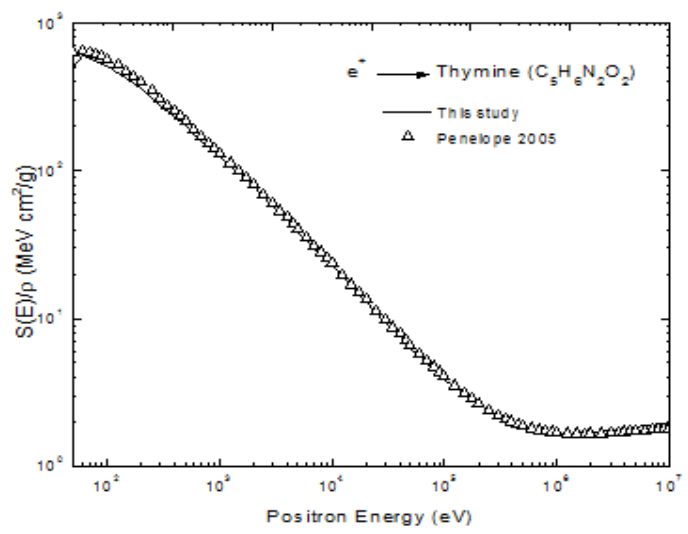

Figure $9 \mathrm{~S}(\mathrm{E}) / \rho$ for incident positron energies, in thymine. - , present study; and $\Delta$, PENELOPE Program ${ }^{27}$ results.

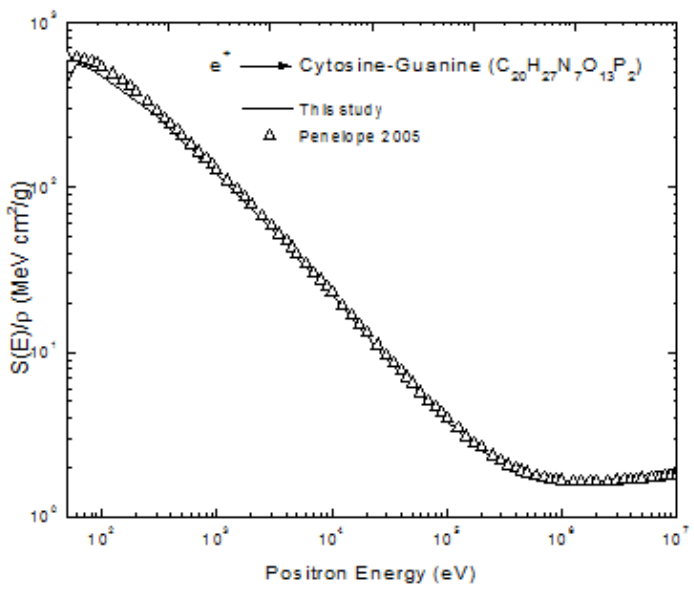

Figure $10 \mathrm{~S}(\mathrm{E}) / \rho$ for incident positron energies, in cytosine-guanine. present study; and $\Delta$, PENELOPE Program ${ }^{27}$ results. 
Figure 12 shows the mSP values for incident positrons from $50 \mathrm{eV}$ to $10 \mathrm{MeV}$ energy range in liquid water. The results obtained in this study are in good agreement with the recommendations of ICRU 37 (1984) for the stopping power of liquid water. The results obtained from this study are compared with the PENELOPE program ${ }^{26}$ and results for positrons by using Gümüş calculation methods. ${ }^{12,}{ }^{28}$ The calculated PSPs values obtained by using Eqs. $(17,18)$ are good agreement with PENELOPE program results to within 3\% above $300 \mathrm{eV}$ energies. The mass stopping powers obtained by using the formalism described in this paper are in good agreement to within data of predictions of Pimblott ${ }^{30}$ above $500 \mathrm{eV}$ energies,

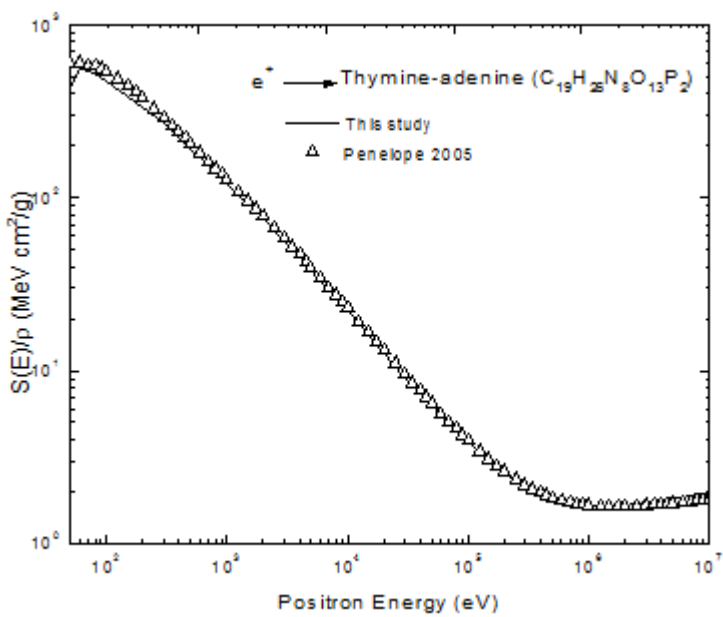

Figure II $\mathrm{S}(\mathrm{E}) / \rho$ for incident positron energies, in thymine-adenine. present study; and $\Delta$, PENELOPE Program ${ }^{27}$ results.

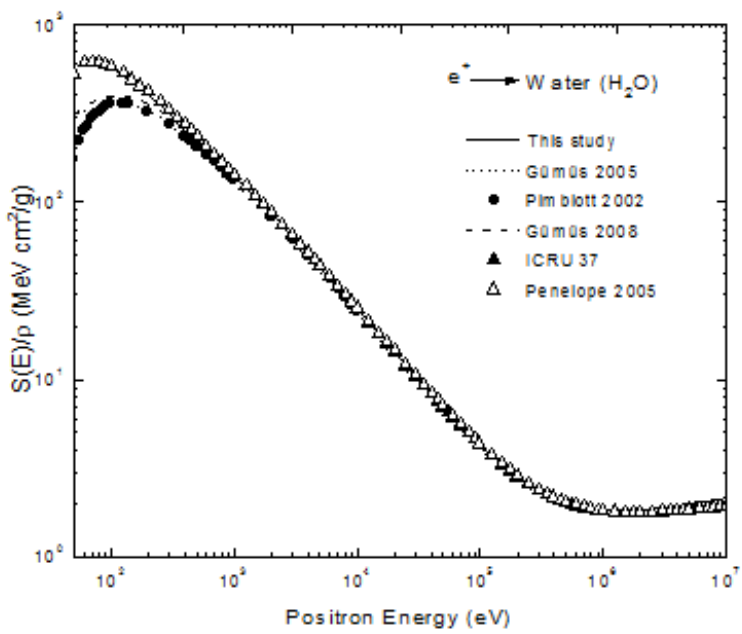

Figure $12 \mathrm{~S}(\mathrm{E}) / \rho$ for incident positron energies, in liquid water. -, present study; and $\Delta$, PENELOPE Program ${ }^{23}$ results. .... theoretical data of Gumus ${ }^{12}$; ---- theoretical data of Gumus ${ }^{29}$; results of Pimblott ${ }^{30}$ and $\boldsymbol{\Delta}$, results of ICRU 37 report. $^{2}$

\section{Concluding remarks}

In this study, the SP values for positrons in biological naterials have been calculated with the GOS model. K shell oscillator strengths, the semiempirical adjustment factor $a, \mathrm{~K}$ and valence shell PMIP values and MIE values of elements have been calculated. In addition, the stopping power for the biological compounds, $\mathrm{C}_{5} \mathrm{H}_{5} \mathrm{~N}_{5}$ (adenine), $\mathrm{C}_{5} \mathrm{H}_{5} \mathrm{~N}_{3} \mathrm{O}$ (guanine), $\mathrm{C}_{4} \mathrm{H}_{5} \mathrm{~N}_{3} \mathrm{O}$ (cytosine), $\mathrm{C}_{20} \mathrm{H}_{27} \mathrm{~N}_{7} \mathrm{O}_{13} \mathrm{P}_{2}$ (cytosineguanine), $\mathrm{C}_{5} \mathrm{H}_{6} \mathrm{~N}_{2} \mathrm{O}_{2}$ (thymine) and $\mathrm{C}_{19} \mathrm{H}_{26} \mathrm{~N}_{8} \mathrm{O}_{13} \mathrm{P}_{2}$ (thymine-adenine)) have been analysed for incident positrons in the $50 \mathrm{eV}-1 \mathrm{MeV}$ energy range. The calculated results of stopping power for positrons in molecules including $\mathrm{H}_{2}, \mathrm{C}, \mathrm{N}_{2}, \mathrm{O}_{2}, \mathrm{C}_{5} \mathrm{H}_{5} \mathrm{~N}_{5} \mathrm{O}$ in biological targets are found to be in good agreement with semiempirical results and theoretical results. The present inelastic SPs calculations for positrons with the GOS model depends on $\mathrm{K}$ and valence shell strengths, $\mathrm{K}$ and valence shell PMIPs. The presented positron SP calculations should be useful for biological target such as $\mathrm{C}_{5} \mathrm{H}_{5} \mathrm{~N}_{5}$ (adenine), $\mathrm{C}_{5} \mathrm{H}_{5} \mathrm{~N}_{3} \mathrm{O}$ (cytosine), $\mathrm{C}_{5} \mathrm{H}_{5} \mathrm{~N}_{5} \mathrm{O}$ (guanine), $\mathrm{C}_{5} \mathrm{H}_{6} \mathrm{~N}_{2} \mathrm{O}_{2}$ (thymine), $\mathrm{C}_{20} \mathrm{H}_{27} \mathrm{~N}_{7} \mathrm{O}_{13} \mathrm{P}_{2}$ (cytosine-guanine) and $\mathrm{C}_{19} \mathrm{H}_{26} \mathrm{~N}_{8} \mathrm{O}_{13} \mathrm{P}_{2}$ (thymine-adenine). In additon, the straggling formula given in this study should be use for biological compounds.

\section{Acknowledgement}

We thank to OECD NEA Computer Program Service (www.nea.fr) for PENELOPE program 2005 and 2008.

\section{Conflict of interest}

The author declares no conflict of interest.

\section{References}

1. Berger MJ, Seltzer SM. Stopping powers and ranges of electrons and positrons. National Bureau of Standarts. 1982;82(2550):1-170.

2. ICRU, Stopping powers for electrons and positrons. International Commission on Radiation Units and Measurements. 1984;37:271.

3. Batra RK. Nucl Instrum Methods. 1987;28:195.

4. Meiring WJ, Van Klinken J, Wichers VA. Rapid Communications Physical Review A. 1991;44(5):2960.

5. Tanir G, Bölükdemir MH, Keleş S. Chinese Journal of Physics. 2012;50(3):425-433.

6. Blanco F, Munoz A, Almeida D, et al. Modelling low energy electron and positron tracks in biologically relevant media. Eur Phys $J$ 2013;67(199):1-18.

7. Gümüş H, Kabadayi Ö, Tufan MC. Calculation of the Stopping Power for Intermediate Energy Positrons. Chinese Journal of Physics. 2006;44(4):290-296

8. Handrack J, Tessonnier T, Chen W, et al. Sensitivity of post treatment positron emission tomography/computed tomography to detect interfractional range variations in scanned ion beam therapy. Acta Oncol. 2017;56(11):1451-1458.

9. Akar A, Gümüș H. Electron stopping power in biological compounds for low and intermediate energies with the generalized oscillator strength (GOS) model. Radiat Phys Chem. 2005;73(4):196-203.

10. Malik E, Juweid MD, Bruce D, et al. Positron-Emission tomography and assessment of cancer therapy. N Engl J Med. 2006;354(5):496-507.

11. Inokuti M. Inelastic Collisions of Fast Charged Particles with Atoms and Molecules-The Bethe Theory Revisited. Rev Mod Phys. 1971;43(3):297.

12. Gümüş $H$. simple stopping power formula for low and intermediate energy electrons. Radiation Physics and Chemistry. 2005;72(1):7-12. 
13. Gümüş H, Kabadayi O, Tufan MC. Calculation of the Stopping Power for Intermediate Energy Positrons. Chnise Journal of Physics. 2006;44(4):290.

14. Bichsel H. Scanning Microsc. Suppl. 1990;4:147.

15. Fernández Varea JM. Rad Phys and Chem. 1998;53:235.

16. Fano U, Ann. Penetration of Protons, Alpha Particles, and Mesons. Rev Nucl Sci. 1963;13:1-16.

17. Inokuti M. Addenda: Inelastic collisions of fast charged particles with atoms and molecules-The Bethe theory revisited. Rev Mod Phys. 1978;50(1):23.

18. Liljequist D. Simple generalized oscillator strength density mode applied to the simulation of $\mathrm{keV}$ electron $\square$ energy $\square$ loss distributions. $J$ Appl Phys. 1985;57(3):657.

19. Bohr N, Mat Fyz. Medd Dan. Vidensk Selsk. 1948;18(8):1.

20. Cengiz A. Approximate inelastic scattering cross sections of electrons Radiation Physics and Chemistry. 2002;65(1):33-44.

21. Henke BL, Gullikson EM, Davis JC. X-Ray interactions: photo absorption, scattering, Transmission and reflection at $\mathrm{E}=50-30,000 \mathrm{eV}$ $\mathrm{Z}=1-92$. Atomic Data and Nuclear Data Tables. 1993;54(2):181-342.
22. Johnson RE, Inokuti M. At Mol Phys. 1983;1419.

23. Berger MJ, Hubbell JH, Seltzer SM, et al. XCOM: Photon Cross Sections Data Base. NIST;1987.

24. Bragg WH, Kleeman R. On the $\alpha$ particles of radium, and their loss of range in passing through various atoms and molecules. Philos Mag.1905;10(57):318-340.

25. Sakurai JJ, Advanced Quantum Mechanics. UK: Addison-Wesley; 1967.

26. Salvat F, Ferandez Varea JM, Sempau. PENELOPE, A Code Systems for monte carlo simulation of electron and photon transport, France: NEA Databank; 2005.

27. Bhabha H. Proc Roy Soc. 1936;154:195.

28. Gümüş H. New stopping power formula for intermideate energy electrons. Applied Radiation and Isotopes. 2008;66(12):1861-1869.

29. Salvat F, Fernández Varea JM. Semiempirical cross sections for the simulation of the energy loss of electrons and positrons in matter. $\mathrm{Nucl}$ Instr And Meth. 1992;63(3):255-269.

30. Pimblott SM, Siebbeles LDA, Nucl Insstr Meth B. 2002;194:237. 